\section{Aufgaben des Betreuungsgerichts}

Dem Betreuungsgericht kommt eine besondere Rolle bei der Kontrolle des Betreuers zu.

Nach SS1908i Abs. 1 S. 1, 1837 Abs. 1, 2 BGB hat das Betreuungsgericht den Betreuer nicht nur zu beraten, sondern auch Aufsicht über die Betreuung zu führen. Der Betreuer ist nach $\int S 1908$ i Abs. 1 S. 1, 1839 BGB verpflichtet, Auskunft über die Führung der Betreuung und die persönlichen Verhältnisse des Betreuten zu erteilen. Gemäß \1840 Abs. 1 S. 2 BGB muss der Bericht eine explizite Stellungnahme zum persönlichen Kontakt zwischen dem Betreuer und den Betroffenen enthalten. Das Betreuungsgericht beaufsichtigt daher auch die Einhaltung des persönlichen Kontakts, \$1837 Abs. 2 S. 2 i. V. mit \$1908i Abs. 1 S. 1 BGB. Klar ist, dass der mangelnde Kontakt zwischen Betreuer und Betroffenen einen Entlassungsgrund darstellt, \1908b Abs. 1 S. 2 Alt. 2 BGB. Schwieriger ist indes, ob sich hieraus auch eine Befugnis ergibt, konkrete Kontakte anzuordnen. Einerseits lehnte dies das LG Nürnberg-Fürth $\mathrm{ab}$, da das Gesetz hierfür keine ausreichende Grundlage biete $^{24}$. Andererseits kann das Betreuungsgericht gegen Pflichtwidrigkeiten des Betreuers mittels geeigneten Geboten und Verboten einschreiten. Eine Pflichtwidrigkeit kann insofern in dem unterlassenen persönlichen Kontakt liegen. In diesem Zusammenhang könnte es dann erlaubt sein, die Einhaltung des regelmäßigen Kontakts - zurzeit auch des telefonischen Kontakts - zu gebieten, $\int \$ 1908 i, 1837$ Abs. 2 S. 1, 3 BGB.

\section{Fazit}

Die Besorgnis, sich mit dem Virus zu infizieren, mag verständlich sein, rechtfertigt aber nicht, auf die Einhaltung sämtlicher verfahrens- und materiell-rechtlicher Vorschriften pauschal und generalisierend zu verzichten. Das Gesetz sieht hierfür hohe Hürden vor. Entscheidend kann daher immer nur der jeweilige Einzelfall sein. Generell gilt, dass der persönliche Kontakt nicht vorschnell abgebrochen werden sollte. Vor allem in Zeiten einer Pandemie können betreute Personen verstärkt auf die Hilfe ihres rechtlichen Betreuers angewiesen sein. Diesen kommt eine wesentliche Rolle sowohl bei der Vorbeugung einer möglichen Ansteckung des Betreuten als auch bei dessen Unterstützung im Falle einer Infektion mit dem Coronavirus zu.

24) LG Nürnberg-Fürth, BtPrax 2015, 50 ff.

\title{
Grundrechte in Zeiten von Corona
}

\author{
Zugleich Anmerkung zu BVerfG, Beschl. v. 7.4.2020 - 1 BvR 755/20

\section{Christian Katzenmeier}

\section{Eilrechtsschutz und Hauptverfahren}

In Zeiten der Corona-Pandemie erfolgen massive Grundrechtseinschränkungen. Deren Verfassungskonformität ist hochstreitig. Bevölkerung wie Fachkreise warten auf einen Richterspruch aus Karlsruhe, der für Rechtsklarheit und Rechtssicherheit sorgt. Die erste inhaltlich begründete Entscheidung des BVerfG erging im Eilverfahren. Nach $\$ 32$ Abs. 1 BVerfGG kann das Gericht eine vorübergehende Anordnung zur Vermeidung schwerwiegender Nachteile erlassen, um irreversible Folgen bis zu einer Entscheidung im Hauptsacheverfahren zu vermeiden. Dabei wendet es eine besondere Prüfungsmethode an: die Folgenabwägung nach der sog. Doppelhypothese. Es werden die Nachteile, die eintreten würden, wenn eine Anordnung des Gerichts nicht erginge, das Hauptverfahren jedoch Erfolg hätte, gegen die Nachteile, die entstünden, wenn die begehrte Anordnung des Gerichts erlassen würde, das Hauptsacheverfahren aber später erfolglos bliebe, abgewogen. Das Ergebnis lautet: „Gegenüber den Gefahren für Leib und Leben wiegen die Einschränkungen der persönlichen Freiheit weniger schwer"1 und so wurde der Antrag auf Erlass einer einstweiligen Anordnung - wie nahezu einhellig erwartet - abgelehnt. Damit ist aber nicht gesagt, dass die weitreichenden Einschränkungen von Grundrechten in der Corona-Krise verfassungsgemäß sind. Die Entscheidung in der Hauptsache kann naturgemäß anders ausfallen als die im Eilrechtsschutz.

Prof. Dr. iur. Christian Katzenmeier,

Universität zu Köln, Institut für Medizinrecht,

Albertus-Magnus-Platz, 50923 Köln, Deutschland

\section{Verfassungsbeschwerde}

1. Formelle Verfassungsmäßigkeit von Kontaktverboten/Ausgangsbeschränkungen

Bei „eingehenderer Prüfung“ der Verfassungsbeschwerde ${ }^{2}$ wird das BVerfG zunächst die formelle Verfassungsmäßigkeit der getroffenen Maßnahmen zu klären haben, im Besonderen die Frage nach der Ermächtigungsgrundlage.

\section{a) IfSG als Ermächtigungsgrundlage}

Um die Corona-Krise aufzufangen, wurde am 25.3.2020 im Bundestag ein ganzes Paket an Gesetzen verabschiedet. Der Bundesrat stimmte in einer Sondersitzung am 27.3. 2020 ohne Aussprache zu, noch am selben Tag wurden die Gesetze im Bundesgesetzblatt verkündet. Zentrales Gesetz zur Bekämpfung der Corona-Epidemie ist das Infektionsschutzgesetz (IfSG), das einige Änderungen erfuhr ${ }^{3}$. Gem. $\ 5$ Abs. 1 S. 1 IfSG kann nun der Deutsche Bundestag eine ,epidemische Lage von nationaler Tragweite" ausrufen. Die aus der epidemischen Lage folgenden Kompetenzen liegen nach $₫ 5$ Abs. 2 IfSG beim Bundesministerium für Gesundheit, das zu weitgehenden Anordnungen und zum Erlass von Rechtsverordnungen ermächtigt wird - ohne Zustimmung des Bundesrates.

1) BVerfG, Beschl. v. 7.4.2020 - 1 BvR 755/20, Rdnr. 11

2) BVerfG, Beschl. v. 7. 4.2020 - 1 BvR 755/20, Rdnr. 7.

3) Durch das ,Gesetz zum Schutz der Bevölkerung bei einer epidemischen Lage von nationaler Tragweite" v. 27.3.2020, BGB1. I S. 587 ff.; dazu Rixen NJW 2020, $1097 \mathrm{ff}$. 
Das IfSG wird von den Bundesländern ausgeführt (Art. 83 GG, $\$ 54$ IfSG), auch bei Ausruf einer epidemischen Lage von nationaler Tragweite ${ }^{4}$. Die in Bayern, Sachsen und im Saarland verhängten Ausgangsbeschränkungen und die in den anderen Bundesländern verordneten Kontaktverbote wurden jeweils auf $\$ 28$ Abs. 1 IfSG gestützt. S. 1 enthält eine Generalklausel, die allgemein zu ,notwendigen Schutzmaßnahmen" ermächtigt, u.a. zur Anordnung individueller Quarantäne (\$30 IfSG), soweit und solange es zur Verhinderung der Verbreitung übertragbarer Krankheiten erforderlich ist. S. 2 erlaubt es den zuständigen Behörden, die in $\$ 33$ IfSG genannten Gemeinschaftseinrichtungen $\mathrm{zu}$ schließen. Nach $\$ 32$ S. 1 IfSG werden die Landesregierungen ermächtigt, unter den Voraussetzungen, die für Maßnahmen nach den $\$ \$ 28$ bis 31 IfSG maßgebend sind, auch durch Rechtsverordnungen entsprechende Gebote und Verbote zur Bekämpfung übertragbarer Krankheiten zu erlassen. Nach S. 2 können die Landesregierungen die Ermächtigung ihrerseits durch Rechtsverordnung auf andere Stellen übertragen ${ }^{5}$.

Mit Wirkung zum 28.3.2020 wurde auch $\$ 28$ Abs. 1 IfSG geändert. Entgegen der Gesetzesbegründung handelt es sich dabei nicht nur um eine Anpassung ,aus Gründen der Normenklarheit" ${ }^{* 6}$ \28 Abs. 1 S. 2 Halbs. 2 IfSG a.F. sah vor, dass Ausgangs- bzw. Betretungsverbote nur so lange möglich waren, ,bis die notwendigen Schutzmaßnahmen durchgeführt worden sind." Die Verschiebung in \$28 Abs. 1 S. 1 Halbs. 2 n. F. unter Streichung dieses Satzendes impliziert, dass Ausgangs- bzw. Betretungsverbote nun zulässig sein sollen, ,solange es zur Verhinderung der Verbreitung übertragbarer Krankheiten erforderlich ist" ${ }^{\text {"7 }}$. Aufenthaltsverbote und -gebote werden nun nicht nur an einzelne, individuell gefährliche Personen gerichtet. Ein allgemeines, von konkret-individuellen Gefahren unabhängiges und in seiner Dauer nicht befristetes Fortbewegungsverbot deckt die Norm aber nicht, auch weil sonst die restriktiven Bestimmungen zur Inanspruchnahme von Nichtstörern unterlaufen werden ${ }^{8}$. Auch wenn mit der Generalklausel eine situationsadäquate Bekämpfung von Infektionskrankheiten ermöglicht werden soll ${ }^{9}$, bei deren Ausbruch die Fülle der Schutzmaßnahmen nicht von vorn herein übersehen werden kann, und auch wenn es sich bei Corona um eine hochdynamische und entwicklungsoffene Krise handelt, so hätte sich der Gesetzgeber bei der Anpassung doch um eine detailliertere Regelung in $\$ 28$ IfSG bemühen müssen. Sollen die weitreichendsten und intensivsten kollektiven Grundrechtseinschränkungen in der Geschichte der Bundesrepublik auf diese Norm gestützt werden, hätte präzisiert werden müssen, welche Maßnahmen unter welchen Voraussetzungen und mit welchen Ausnahmen angeordnet werden dürfen ${ }^{10}$. So aber ,,spricht wenig dafür, dass eine Stilllegung des gesamten öffentlichen Lebens (...) durch das IfSG ermöglicht werden sollte. Die dagegen vertretene Ansicht, das Land ließe sich mit Hilfe einer Generalklausel dicht machen, erscheint einigermaßen kurios. Sie macht aus einem besonderen Polizeirecht ein allgemeines Notstandsrecht" ${ }^{\text {"11 }}$.

Hochproblematisch ist zudem die Regelung in $\$ 5$ Abs. 2 Nr. 3 IfSG, wonach der Bundesgesundheitsminister durch Rechtsverordnung ohne Zustimmung des Bundesrates von gesetzlichen Vorschriften des IfSG abweichen darf ${ }^{12}$. Das ist mit Art. 80 Abs. 1 S. 2 GG unvereinbar ${ }^{13}$. Die parlamentarischen Befugnisse werden über das verfassungsrechtlich zulässige $M a ß$ hinaus auf die Exekutive verlagert. Dabei kommt dem Bundestag als dem zentralen demokratischen Verfassungsorgan in dieser Situation eine besondere Bedeutung zu. Denn demokratische politische Entscheidungen setzen Öffentlichkeit und Diskussion voraus. Das Parlament ist das institutionelle Kernstück dieser demokratischen Idee ${ }^{14}$. Die (Selbst-) Entmachtung der Legislative in der Corona-Krise zugunsten der Exekutive gefährdet die Gewaltenteilung ${ }^{15}$.

\section{b) Wesentlichkeitslehre}

Nach der Wesentlichkeitslehre muss der Gesetzgeber ,in grundlegenden normativen Bereichen, zumal im Bereich der Grundrechtsausübung, soweit diese staatlicher Regelung zugänglich ist, alle wesentlichen Entscheidungen selbst (...) treffen" $"$, das heißt er darf sie nicht auf die Verwaltung delegieren. Der Gesetzesvorbehalt erstarkt insoweit zum Parlamentsvorbehalt. Die Wesentlichkeit der Entscheidung bemisst sich nach der Intensität, in der Grundrechte betroffen sind. Auch die Regelungsdichte bemisst sich danach. Je intensiver Grundrechte betroffen sind, desto genauer und differenzierter muss das Parlamentsgesetz sein ${ }^{17}$

In der Corona-Krise fehlt es nach wie vor an hinreichend bestimmten Parlamentsgesetzen, die derart weitreichende kollektive Grundrechtsbeeinträchtigungen, insbes. der

4) Die Ermächtigung des BMG nach $₫ 5$ Abs. 2 IfSG erfolgt „unbeschadet der Befugnisse der Länder".

5) Eine Übertragung auf das Landesgesundheitsministerium erfolgt etwa nach $\$ 10$ ZVO-IfSG NRW.

6) BT-Dr. $19 / 18111$ v. 24.3.2020, S. 25: Begr. zu Art. 1 Nr. 6 ( $\$ 28$ IfSG).

7) Zur Frage, inwieweit die Änderungen des IfSG bereits vor dem 28.3.2020 erlassene rechtswidrige Verordnungen legitimieren können vgl. Klafki, Neue Rechtsgrundlagen im Kampf gegen Covid-19: Der Gesetzesentwurf zum Schutz der Bevölkerung bei einer epidemischen Lage von nationaler Tragweite, VerfBlog, 2020/3/25, https://verfassungsblog.de/neue-rechtsgrundlagen-im-kampf-gegen-covid-19/.

8) Kingreen, Whatever it Takes?: Der demokratische Rechtsstaat in Zeiten von Corona, VerfBlog, 2020/3/20, https://verfassungsblog.de/whatever-it-takes/. Dies gilt, auch wenn das IfSG im Unterschied zum allgemeinen Gefahrenabwehrrecht auf der Basis sehr niederschwelliger Verdachtsgrade operiert und stärker bereits an den Gefahrenverdacht anknüpft, dazu Rixen. NJW 2020, 1097, zur mgl. Inpflichtnahme von Nichtstörern 1101.

9) Vgl. BT-Dr. 14/2530, S. 74f., der Gesetzgeber des IfSG hat sich an diesen Wertungen des früheren BSeuchG angelehnt.

10) Kießling, Ausgangssperren wegen Corona nun auch in Deutschland(?), JuWissBlog Nr. 29/2020 v. 19.3.2020, https://www. juwiss.de/29-2020/; Klafki, Neue Rechtsgrundlagen im Kampf gegen Covid-19: Der Gesetzesentwurf zum Schutz der Bevölkerung bei einer epidemischen Lage von nationaler Tragweite, VerfBlog, 2020/3/25, https://verfassungsblog.de/neue-rechtsgrundlagen-im-kampf-gegen-covid-19/; großzügiger Rixen, NJW 2020, 1097, 1099.

11) Möllers, Parlamentarische Selbstentmächtigung im Zeichen des Virus, VerfBlog, 2020/3/26, https://verfassungsblog.de/parlamentarische-selbstentmaechtigung-im-zeichen-des-virus/.

12) Ursprünglich sollte sogar die Kompetenz zur Feststellung der gesundheitlichen Notlage nach $\ 5$ Abs. 1 IfSG beim BMG liegen.

13) Näher Rixen, NJW 2020, 1097, 1102f. m.w. N.

14) Schönberger, Die Stunde der Politik, VerfBlog, 2020/3/29, https:// verfassungsblog.de/die-stunde-der-politik/; Thielbörger/Behlert, COVID-19 und das Grundgesetz: Neue Gedanken vor dem Hintergrund neuer Gesetze, VerfBlog, 2020/3/30, https://verfassungsblog.de/covid-19-und-das-grundgesetz-neue-gedanken-vor-dem-hintergrund-neuer-gesetze/ erachten eine Verankerung der Regelungen im GG für notwendig.

15) Möllers, Parlamentarische Selbstentmächtigung im Zeichen des Virus, VerfBlog, 2020/3/26, https://verfassungsblog.de/parlamentarische-selbstentmaechtigung-im-zeichen-des-virus/; Lepsius, Vom Niedergang grundrechtlicher Denkkategorien in der Corona-Pandemie, VerfBlog, 2020/4/06, https://verfassungsblog.de/vom-niedergang-grundrechtlicher-denkkategorien-in-der-corona-pandemie/: ,Wenn schon auf dem Verordnungswege entschieden wird, dann wäre es angesichts der grundrechtlichen Relevanz zwingend erforderlich, dass die Regierungen entscheiden (...). Auch organisationsrechtlich ist momentan nichts falscher als Gesundheitsminister mit einem Sonderverordnungsrecht auszustatten."

16) BVerfGE $61,260,275 ; 88,103,116 ; 133,112,132$; Kingreen Poscher, Grundrechte, 35. Aufl. 2019, Rdnr. 315.

17) BVerfGE 49,168, 181; 59, 104, 114; 86, 288, 311; Maurer, Staatsrecht, 6 . Aufl. 2020, $\$ 8$, Rdnrn. 21 f.; Kingreen/Poscher, Grundrechte, 35. Aufl. 2019, Rdnrn. $317 \mathrm{ff}$. 
Freiheit der Person (Art. 2 Abs. 2 GG), der Versammlungsfreiheit (Art. 8 GG), der Freizügigkeit (Art. 11 Abs. 1 GG), der Berufsausübungsfreiheit (Art. 12 Abs. 1 GG) und der Religionsausübungsfreiheit (Art. 4 GG) durch Exekutivgesetze vorsehen. Dabei ist dieser Legalitätsgrundsatz „,keine formalistische Petitesse, die man wegen drängenderer Probleme einfach beiseiteschieben kann“, er zählt vielmehr zum Fundament unseres Rechtsstaats ${ }^{18}$.

\section{c) „Notstandsvorbehalt“?}

Die Judikatur erkennt allerdings an, dass es im Rahmen unvorhergesehener Entwicklungen aus übergeordneten Gründen des Gemeinwohls geboten sein kann, nicht hinnehmbare gravierende Regelungslücken für einen Übergangszeitraum insbesondere auf der Grundlage von Generalklauseln zu schließen und auf diese Weise selbst sehr eingriffsintensive Maßnahmen, die an sich einer besonderen Regelung bedürfen, vorübergehend zu ermöglichen ${ }^{19}$. Dem hält Christoph Möllers entgegen: „Sollten wir aus der Krise mit der Einsicht herausgehen, dass fundamentale Normen der Arbeitsteilung zwischen Parlament und Regierung wie zwischen Bund und Ländern befristet unter einem ungeschriebenen verfassungsrechtlichen Notstandsvorbehalt stehen, wäre das fatal" ${ }^{20}$

Das BVerfG geht in seinem Beschluss v. 7.4.2020 auf das Legalitätsprinzip nicht ein, weil Gegenstand des Eilverfahrens bei offenem Ausgang der Verfassungsbeschwerde lediglich die Folgenabwägung nach der Doppelhypothese ist, nicht aber sind die Erfolgsaussichten in der Hauptsache zu bewerten. Anders verfährt das Gericht nur, wenn die Verfassungsbeschwerde offensichtlich unzulässig oder unbegründet ist ${ }^{21}$, was vorliegend ausdrücklich verneint wurde ${ }^{22}$.

\section{Materielle Verfassungsmäßigkeit der Grundrechtsbeschränkungen}

Die materielle Verfassungsmäßigkeit der Grundrechtsbeschränkungen in der Corona-Krise ist nicht einfach zu beurteilen. Als die Maßnahmen Mitte März 2020 verordnet wurden, war das Leben von Unsicherheit geprägt, auch in Deutschland wurden dramatische Zustände befürchtet. Wenn in einer existentiellen Krise der Staat nicht entschlossen handelt, kann in der Bevölkerung schnell der Eindruck von Hilf-/Verantwortungslosigkeit entstehen, die Verunsicherung wachsen und in Panik umschlagen. So ist es im Ausgangspunkt durchaus nachvollziehbar, dass zum Zwecke der Eindämmung der Pandemie drastische Maßnahmen ergriffen wurden.

\section{a) Abwägung - Zulässigkeit und Notwendigkeit}

Doch auch wenn der Gesetzgeber bei der schwierigen Beurteilung komplexer Zusammenhänge und insbesondere be Gefährdungslagen für Leib und Leben eine weite Einschätzungsprärogative für sich in Anspruch nehmen $\mathrm{kann}^{23}$, auch wenn die jüngere Hinwendung zum Präventionsstaat zunehmend darauf abzielt, Krisen möglichst im Vorfeld zu antizipieren, also nicht erst dann aktiv zu werden, wenn eine konkrete Gefahr besteht, sondern schon die Situation zu verhindern, aus der heraus sie womöglich irgendwann entstehen könnte ${ }^{24}$, und auch wenn in der Bevölkerung ,eine gewisse Neigung besteht, nicht nur die sinnvollen Maßnahmen mitzutragen, sondern auch darüber hinauszugehen", eine Mentalität zu beobachten ist, ,sich einer verstärkten Daseinsvorsorge in jeder Hinsicht auszuliefern "25, ist in Zeiten nationalen Notstands die Rechtfertigung des Grundrechtseingriffs nicht etwa entbehrlich, sondern bleibt zentrale Frage ${ }^{26}$.

In Anbetracht der in der Krise hierzulande gut funktionierenden staatlichen Institutionen mögen Befürchtungen übertrieben sein, unser Gemeinwesen könne sich in kürzester Frist von einem demokratischen Rechtsstaat in einen „faschistoid-hysterischen Hygienestaat“ verwandeln ${ }^{27}$. Doch besteht im „Ausnahmezustand“ unverkennbar der Drang, zum Schutze aller auch die Rechte aller einzuschränken. Grundrechte werden dann weniger in ihrer klassischen Funktion als Abwehrrechte gegenüber dem Staat gesehen, stattdessen wird die aus ihnen fließende staatliche Schutzpflicht betont. Die Aufmerksamkeit gilt weniger dem Übermaß- denn dem Untermaßverbot. Sicherheit siegt über Freiheit $^{28}$. In der Corona-Krise kulminiert dies in der-weitestgehend akzeptierten - Feststellung: ,Gegenüber den Gefahren für Leib und Leben wiegen die Einschränkungen der persönlichen Freiheit weniger schwer" 29 .

Dieser Satz stellt jedoch - jenseits des Eilrechtsschutzverfahrens - nicht nur eine unzulässige Verkürzung dar, er droht jeden Diskurs bereits im Keime zu ersticken. Dass am Ende immer die Bedeutung der auf dem Spiel stehenden Rechtsgüter und die schiere Größe der Gefahr auch ganz drastische Maßnahmen rechtfertigen, ist so richtig wie trivial ${ }^{30}$. Um eine sachliche Diskussion $\mathrm{zu}$ ermöglichen, haben Thorsten Kingreen, Uwe Volkmann und Stefan Huster früh darauf hingewiesen, dass eine abwägungsblinde Verabsolutierung des Lebensschutzes, eine Politik der maximalen Bekämpfung des Corona-Virus um jeden Preis, grundrechtlich nicht der richtige Weg sein kann ${ }^{31}$. Seit jeher verzeichnen wir jährlich tausende Straßenverkehrstote um der Mobilität willen, Grippe-Tote, ohne dass ein Impfzwang

18) DAV, Stellungnahme Nr. 22/2020 v. 23.3.2020 durch den Ausschuss Gefahrenabwehrrecht; Bäcker, Corona in Karlsruhe II, VerfBlog, 2020/4/08, https://verfassungsblog.de/corona-inkarlsruhe-ii/.

19) So im vorliegenden Kontext OVG NRW, Beschl. v. 6.4.2020 - 13 B 398/20.NE, Rdnr. 59; s. auch allg. BVerwGE 164, $304=$ NVwZ 2019, 635; BVerfG, DVBl. 2013, 169.

20) Möllers, Parlamentarische Selbstentmächtigung im Zeichen des Virus, VerfBlog, 2020/3/26, https://verfassungsblog.de/parlamentarische-selbstentmaechtigung-im-zeichen-des-virus/.

21) St. Rspr., vgl. etwa BVerfGE 112, 284, 291; 121, 1, $14 \mathrm{f}$.

22) BVerfG, Beschl. v. 7. 4.2020-1 BvR 755/20, Rdnr. 7. Für den Erlass einer einstweiligen Anordnung wegen offensichtlichen Erfolgs der Hauptsache wegen kaum zu bestreitender formeller Verfassungswidrigkeit plädiert Bäcker, Corona in Karlsruhe II, VerfBlog, 2020/4/08, https://verfassungsblog.de/corona-in-karlsruhe-ii/; s. dazu allg. BeckOK-BVerfGG/Walter, 8. Ed. 1.1.2020, \$32, Rdnrn. $58 \mathrm{ff}$.

23) S. nur etwa BVerfGE 39, 1; 88, 203, 262; Kingreen/Poscher, Grundrechte, 35. Aufl. 2019, Rdnr. $133 \mathrm{ff}$

24) Volkmann, Der Ausnahmezustand, VerfBlog, 2020/3/20, https:// verfassungsblog.de/der-ausnahmezustand/; näher Denninger, Der Präventions-Staat, KJ 1988, 1 ff.; ders., Der gebändigte Leviathan, 1990; ders., Recht in globaler Unordnung, 2005, Kap. 14; Grimm, Die Zukunft der Verfassung, 1991; Di Fabio, Risikoentscheidungen im Rechtsstaat, 1993, \$16 A; Albrecht, Vom Präventionsstaat zur Sicherheitsgesellschaft - Wege kontinuierlicher Erosion des Rechts, in: FS f. Hassemer, 2010, S. 3 ff.

25) Gerhart Baum, Interview Legal Tribune Online v. 11. 4. 2020.

26) Blick auf die Geschichte des nationalen Notstands von Lemke, Die Zeit v. 26.3.2020, S. 17: In existentiellen Krisen ist ein starker Staat unverzichtbar. Doch wann wird die Einschränkung von Grundrechten zur Gefahr für die Demokratie?

27) Heinig, Gottesdienstverbot auf Grundlage des Infektionsschutzgesetzes: Verfassungsrechtliche Rechtfertigung und Grenzen, VerfBlog, 2020/3/17, https://verfassungsblog.de/gottesdienstverbot-auf-grundlage-des-infektionsschutzgesetzes/.

28) Scharfe Kritik übt Lepsius, Vom Niedergang grundrechtlicher Denkkategorien in der Corona-Pandemie, VerfBlog, 2020/4/06, https://verfassungsblog.de/vom-niedergang-grundrechtlicherdenkkategorien-in-der-corona-pandemie/.

29) Er findet sich auch in BVerfG, Beschl. v. 7.4.2020 - 1 BvR 755/20, Rdnr. 11

30) Volkmann, Der Ausnahmezustand, VerfBlog, 2020/3/20, https:// verfassungsblog.de/der-ausnahmezustand/.

31) Kingreen, Whatever it Takes?: Der demokratische Rechtsstaat in Zeiten von Corona, VerfBlog, 2020/3/20, https://verfassungsblog.de/whatever-it-takes/; Volkmann, Der Ausnahmezustand, VerfBlog, 2020/3/20, https://verfassungsblog.de/der-ausnahmezustand/; Huster, Grenzen der Solidarität, VerfBlog, 2020/3/22, https://verfassungsblog.de/grenzen-der-solidaritaet/. 
eingeführt würde, Tote auf der Warteliste, weil wir die Freiheit zum Entschluss einer postmortalen Organspende respektieren. Der grundrechtliche Lebensschutz ist in ein Verhältnis zu setzen zur freien Entfaltung der Persönlichkeit, zu anderen grundrechtlich geschützten Freiheiten und zu sonstigen Gütern von Verfassungsrang ${ }^{32}$.

Die eigentliche Herausforderung durch die CoronaKrise sieht Stefan Huster dementsprechend nicht im Sterben als solchem, sondern in den Rahmenbedingungen und Begleiterscheinungen, unter denen sich dieses Sterben vollzieht ${ }^{33}$. Er verweist auf die drohende Notwendigkeit von Triage-Entscheidungen, also Entscheidungen darüber, wer ein Beatmungsgerät erhält und wer nicht ${ }^{34}$, und auf Bilder von überfordertem Klinikpersonal, die auch für den Betrachter unerträglich scheinen. Ziel aller staatlicher Maßnahmen zur Eindämmung der Epidemie ist es, die Infektionskurve abzuflachen, damit nicht zu viele Fälle auf einmal die Krankenhäuser fluten, mithin die Vermeidung der Überforderung des Gesundheitssystems durch optimale Steuerung des Pandemieverlaufs am Maßstab der medizinischen Kapazität. Lebensschutz ist dabei mittelbarer Zweck, nicht aber das direkte Ziel der Eingriffe ${ }^{35}$.

\section{b) Verhältnismäßigkeit}

Bei der Statuierung der notwendigen Ermächtigungsgrundlagen ist der Gesetzgeber an die Grundrechte gebunden, Art. 1 Abs. 3 GG. Konsequent aktualisiert das BVerfG diese Bindung in der Kontrolle der Gesetzgebung auf ihre Verhältnismäßigkeit ${ }^{36}$. Grundrechtseingreifende gesetzliche Regelungen und Ermächtigungen halten einer verfassungsrechtlichen Überprüfung nur stand, wenn sie zur Erreichung des jeweils verfolgten, seinerseits verfassungslegitimen Zwecks geeignet, erforderlich und angemessen sind. Die Beschränkungen individueller Freiheiten sind mit dem erwarteten Nutzen der getroffenen Maßnahmen zur Bekämpfung der Epidemie in ein ausgewogenes Verhältnis zu bringen ${ }^{37}$.

\section{aa) Geeignetheit}

Hinsichtlich der Geeignetheit der ergriffenen Maßnahmen hat sich die Politik hierzulande von Beginn an durch Virologen und Epidemiologen sachverständig beraten lassen. Maßgebliche Bedeutung kommt den Risikobewertungen durch das Robert Koch-Institut (RKI) zu, nach \$4 IfSG nationale Behörde zur Vorbeugung übertragbarer Krankheiten sowie zur frühzeitigen Erkennung und Verhinderung der Weiterverbreitung von Infektionen. Die Zuständigkeit dieser Bundesoberbehörde im Geschäftsbereich des Bundesministeriums für Gesundheit schließt die Entwicklung und Durchführung epidemiologischer und laborgestützter Analysen sowie Forschung zu Ursache, Diagnostik und Prävention übertragbarer Krankheiten ein. Das RKI arbeitet mit den jeweils zuständigen Bundesbehörden, den zuständigen Landesbehörden, den nationalen Referenzzentren, weiteren wissenschaftlichen Einrichtungen und Fachgesellschaften zusammen. Wissenschaftler (hier: Virologen und Epidemiologen) liefern Tatsachen; Wertungen und Schlussfolgerungen sind Sache der demokratisch legitimierten Institutionen.

Bei der Atemwegserkrankung Covid-19 stellte sich das Problem des Fehlens naturwissenschaftlicher Bewertungen und Prognosen der Krankheitsentwicklung. Wie in all diesen Fällen, in denen eine komplexe Gefahrenprognose erforderlich ist, wird man - wenn überhaupt - erst hinterher wissen, welche Maßnahmen angezeigt waren ${ }^{38}$. Der Gesetzgeber kann in solchen hochdynamischen und entwicklungsoffenen Prozessen stets nur ,,auf Sicht fahren“39.

bb) Erforderlichkeit

Kritisch zu hinterfragen ist die Erforderlichkeit einer jeden Grundrechtsbeschränkung. Erforderlich ist eine Maß- nahme, wenn der verfolgte Zweck nicht durch ein gleich wirksames, aber weniger belastendes Mittel erreichbar ist ${ }^{40}$. Eine Kontaktsperre, die Kontakte zu anderen Menschen auBerhalb der Angehörigen des eigenen Hausstands auf ein absolut nötiges Minimum reduzieren soll, ist ein schwerwiegender staatlicher Eingriff in die Freiheitsrechte des Einzelnen. Es stellt sich die Frage, ob die Abflachung der Infektionskurve durch Einhaltung des gebotenen Abstands $\mathrm{zu}$ anderen Menschen nicht ebenso durch einen Appell an das Verantwortungsbewusstsein der Bevölkerung erreichbar ist - welches von Gesetzes wegen verdeutlicht und gefördert werden soll, $₫ 1$ Abs. 2 IfSG. Von diesem milderen Mittel versprach sich die Politik indes keine gleiche Wirksamkeit, da uneinsichtige Zeitgenossen sich nicht durch Vernunft, sondern nur durch Verbote vom Besuch von Clubs und Kinos oder gar Corona-Partys abhalten ließen ${ }^{41}$.

32) Insoweit auch Kube, Leben in Würde - Würde des Lebens, VerfBlog, 2020/4/02, https://verfassungsblog.de/leben-in-wuerdewuerde-des-lebens/: Unverfügbar ist allein die Menschenwürde. Das höchste Gut, auf das sich die politische Gemeinschaft verpflichtet hat, ist deshalb nicht das Leben, sondern - wie es Uwe Volkmann formuliert - das „Leben in Würde“.

33) Huster, Grenzen der Solidarität, VerfBlog, 2020/3/22, https:// verfassungsblog.de/grenzen-der-solidaritaet/.

34) $\mathrm{Zu}$ den Schwierigkeiten der Entwicklung von Entscheidungskriterien für Triage-Situationen s. die Ad-hoc Empfehlung des Deutschen Ethikrats, Solidarität und Verantwortung in der Corona-Krise, und die Beiträge von Buүx/Lipp, Lübbe, Taupitz in diesem Heft.

35) Lepsius, Vom Niedergang grundrechtlicher Denkkategorien in der Corona-Pandemie, VerfBlog, 2020/4/06, https://verfassungsblog.de/vom-niedergang-grundrechtlicher-denkkategorien-inder-corona-pandemie/.

36) BVerfGE 75, 108, 154f.; 80, 137, 153; 90, 145, 172; 96, 10, 21; 97 , 271, 286; zum Vorbehalt des verhältnismäßigen Gesetzes Schlink, EuGRZ 1984, 457, 459f.; ders., in: FS 50 Jahre BVerfG, Bd. II, 2001, S. 445; Kingreen/Poscher, Grundrechte, 35. Aufl. 2019, Rdnrn. 321 ff.; Dreier, in: Merten/Papier, Handbuch der Grundrechte in Deutschland und Europa, Bd. 1, 2004, $\$ 4$, Rdnr. 34

37) Thielbörger/Behlert, COVID-19 und das Grundgesetz: Neue Gedanken vor dem Hintergrund neuer Gesetze, VerfBlog, 2020/3/30, https://verfassungsblog.de/covid-19-und-das-grundgesetz-neue-gedanken-vor-dem-hintergrund-neuer-gesetze/

38) Volkmann, Der Ausnahmezustand, VerfBlog, 2020/3/20, https:// verfassungsblog.de/der-ausnahmezustand/: Wir werden erst hinterher wissen, ob wir richtig gehandelt haben, ob man viel früher oder viel härter hätte handeln müssen oder ob das Ganze nicht doch ein „Feuerwerk des Wahnsinns" war.

39) So entschied auch der BayVerfGH v. 26.3.2020 - Vf. 6-VII20 ausdrücklich ,im gegenwärtigen Stadium des Verfahrens“. Schönberger, Die "Stunde der Politik, VerfBlog, 2020/3/29, https://verfassungsblog.de/die-stunde-der-politik/: „Da es sich um einen neuen Virus handelt, zu dem die Forschung noch ganz am Anfang steht, können uns die Virologen und Epidemiologen keine verbindlichen, über alle Zweifel erhabenen wissenschaftlichen Handlungsanweisungen geben - und kommunizieren dies zum Glück auch offen. Das bedeutet für uns als demokratische Gemeinschaft, dass wir wieder lernen müssen, mit den Unsicherheiten und Ungewissheiten demokratischer Entscheidungen zu leben, die jetzt trotz dieser wissenschaftlichen Uneindeutigkeiten getroffen werden müssen."

40) Kingreen/Poscher, Grundrechte, 35. Aufl. 2019, Rdnr. 336.

41) Kingreen, Whatever it Takes?: Der demokratische Rechtsstaat in Zeiten von Corona, VerfBlog, 2020/3/20, https://verfassungsblog.de/whatever-it-takes/; Zweifel an der Aufopferungspflicht zugunsten Dritter äußert Huster, Grenzen der Solidarität, VerfBlog, 2020/3/22, https://verfassungsblog.de/grenzen-der-solidaritaet/; demgegenüber betont Kube, Leben in Würde - Würde des Lebens, VerfBlog, 2020/4/02, https://verfassungsblog.de/ leben-in-wuerde-wuerde-des-lebens/ Gemeinsinn und generationsübergreifende Solidarität. Dem Verlangen nach freier und gleicher Persönlichkeitsentfaltung in Würde stehe ,sehr deutlich und grundrechtsintensiv die Würde des Lebens der in besonderer Weise gefährdeten Älteren und Schwachen gegenüber, die es zu wahren gilt." 
Bei Ausgangsbeschränkungen, wie sie in Bayern verhängt wurden $^{42}$, dürfen die Bürger darüber hinaus die eigene Wohnung nur bei Vorliegen eines triftigen Grundes verlassen. Die Politik setzt auf den disziplinierenden Charakter der Maßnahme ${ }^{43}$, es ist jedoch nicht ersichtlich, dass dadurch der Pandemieverlauf am Maßstab der medizinischen Kapazitäten besser gesteuert würde als durch ein Kontaktverbot. Damit ist diese Grundrechtseinschränkung unverhältnismäßig, auch wenn Ausnahmen zugelassen werden. Die Bürger dürfen nicht gezwungen werden, sich gegenüber der Polizei zu rechtfertigen, warum sie von grundlegenden Freiheiten Gebrauch machen ${ }^{44}$. Liegen keine gesicherten wissenschaftlichen Erkenntnisse und Handlungsanweisungen vor, ist es umso wichtiger, Grundrechtseingriffe strikt zu begrenzen in der Hoffnung, dass mit der Zeit Tatsachen und Erfahrungen verfügbar sind, die dann überprüfbare Prognosen ermöglichen ${ }^{45}$.

cc) Rückkehr zur Normalität

Besonders betont sei, dass der Grundsatz der Verhältnismäßigkeit auch eine zeitliche Komponente hat. Je länger die Einschränkungen dauern, desto höher sind die Anforderungen an ihre Rechtfertigung ${ }^{46}$. Ein Mehr an Gesundheitsschutz führt zu längeren Freiheitsverlusten und darüber zu größerem Schaden des sozialen Lebens, des kulturellen Lebens und nicht zuletzt der Wirtschaft, zu Schaden für Arbeitsplätze, die soziale Sicherheit, für Rentensysteme und Steuerkassen, zu Armut - mit ihrerseits nachteiligen Auswirkungen auf die Gesundheit ${ }^{47}$. „Niemand will aus dem gegenwärtigen Alptraum in einem Trümmerfeld erwachen, in dem ganze Wirtschaftszweige, eine Vielzahl von Unternehmen und massenhaft individuelle berufliche Existenzen vernichtet sind" 48 .

Erklärtes Ziel muss die schnellstmögliche Rückkehr zur Normalität sein, aber nicht die „Normalität“ einer neuen, exekutiv-mächtigeren Verfassung. Sämtliche grundrechtsbeschränkende Maßnahmen sind vom Parlament permanent zu überprüfen. Bei länger andauernder Sondersituation ist eine zunehmend kritische Distanz notwendig, um zu verhindern, dass der „Ausnahmezustand“ schleichend zur Normallage wird ${ }^{49}$. Krisenregelungen dürfen nicht so beschaffen sein, dass sie Machterweiterung und -zentralisierung auf Dauer zulassen ${ }^{50}$. „Infektiös ist nicht nur das Corona-Virus, sondern auch die autoritäre Versuchung" ${ }^{51}$.

Unabdingbar ist schließlich, die politischen Entscheidungen in einer offenen Demokratie transparent zu machen und zu erläutern, zu begründen und zu kommunizieren. Keinesfalls reichen Pauschalbehauptungen, die alsbald in Kraft tretende Maßnahme sei notwendig ${ }^{52}$. Ebenso ist über den Fortbestand und die Dauer von Grundrechtseinschränkungen in der Demokratie ein offener Diskurs zu führen. Es braucht Augenmaß und Verantwortungsbewusstsein - auf Seiten der Regierung wie auf Seiten der Bevölkerung. Eine risikoadaptierte Strategie führt aus der Krise ${ }^{53}$. Mit Blick auf unsere gesamte Demokratie müssen wir immer bedenken: „Die Medizin darf nicht gefährlicher sein als die Krankheit" ${ }^{\text {" }}$.

42) Vollzug des IfSG: Vorläufige Ausgangsbeschränkung anlässlich der Corona-Pandemie. Bekanntmachung des Bayerischen Staatsministeriums für Gesundheit und Pflege v. 20.3.2020, BayMB1. Nr. 152, 4.: „Das Verlassen der eigenen Wohnung ist nur bei Vorliegen triftiger Gründe erlaubt"; ebenso in Sachsen und im Saarland.

43) Burger et al., faz.net v. 22.3.2020, abrufbar unter https://www. faz.net/aktuell/politik/inland/corona-krise-kontaktsperre-oderausgehverbot-16691693-p2.html.

44) So auch DAV, Stellungnahme Nr. $22 / 2020$ v. 23.3.2020, Ausschuss Gefahrenabwehrrecht.

45) Lepsius, Vom Niedergang grundrechtlicher Denkkategorien in der Corona-Pandemie, VerfBlog, 2020/4/06, https://verfassungsblog.de/vom-niedergang-grundrechtlicher-denkkategorien-in-der-corona-pandemie/.

46) Kingreen, Whatever it Takes?: Der demokratische Rechtsstaat in Zeiten von Corona, VerfBlog, 2020/3/20, https://verfassungsblog.de/whatever-it-takes/.

47) Nienhaus, Die Zeit v. 26.3.2020, S. 23

48) Volkmann, Der Ausnahmezustand, VerfBlog, 2020/3/20, https:// verfassungsblog.de/der-ausnahmezustand/; Spamann, Beispiellose Freiheitseingriffe brauchen beispiellose Transparenz, VerfBlog, 2020/4/04, https://verfassungsblog.de/beispiellose-freiheitseingriffe-brauchen-beispiellose-transparenz/ beanstandet, dass zu ökonomischen und anderen Folgeabschätzungen nichts gesagt wird. „Präzision ist weder möglich noch erforderlich. Aber Wahrscheinlichkeitsabschätzungen oder wenigstens Möglichkeitsanalysen sind für verantwortliches Handeln schlechterdings unabdingbar. Je nach Informationslage kann die gleiche Maßnahme angemessen, unzureichend, oder übertrieben sein."

49) Schönberger, Die Stunde der Politik, VerfBlog, 2020/3/29, https:// verfassungsblog.de/die-stunde-der-politik/.

50) Thielbörger/Behlert, COVID-19 und das Grundgesetz: Neue Gedanken vor dem Hintergrund neuer Gesetze, VerfBlog, 2020/3/30, https://verfassungsblog.de/covid-19-und-das-grundgesetz-neue-gedanken-vor-dem-hintergrund-neuer-gesetze/. In Nordrhein-Westfalen hat der Landtag das sog. Epidemie-Gesetz nach heftiger Kritik entschärft. Die ,,epidemische Lage von landesweiter Tragweite" läuft nun alle zwei Monate aus, es sei denn, der Landtag verlängert sie. Die Verordnungsermächtigungen des Bundes in $\$ 5$ Abs. 2 IfSG gehen weit über das hinaus, vgl. $\$ 5$ Abs. 4 IfSG: ohne Aufhebung der Feststellung der epidemischen Lage von nationaler Tragweite erst mit Ablauf des 31.3.2021.

51) Lemke, Die Zeit v. 26.3.2020, S. 17; s. auch Blog „Demokratie im Ausnahmezustand“, abrufbar unter https://emergency.hypotheses.org/6665; Lepsius, Vom Niedergang grundrechtlicher Denkkategorien in der Corona-Pandemie, VerfBlog, 2020/4/06, https:// verfassungsblog.de/vom-niedergang-grundrechtlicher-denkkategorien-in-der-corona-pandemie/: „Der Rechtsstaat ist schwer beschmutzt. Die rechtsstaatliche Hygiene muss dringend wieder hergestellt werden, sonst droht hier das größte Infektionsrisiko."

52) Spamann, Beispiellose Freiheitseingriffe brauchen beispiellose Transparenz, VerfBlog, 2020/4/04, https://verfassungsblog.de/ beispiellose-freiheitseingriffe-brauchen-beispiellose-transparenz/.

53) S. dazu die Empfehlung des Expertenrats Corona „Die Bekämpfung der Coronavirus-Pandemie tragfähig gestalten“, abrufbar unter https://www.ifo.de/DocDL/Coronavirus-Pandemie-Strategie-Fuest-Lohse-etal-2020-04.pdf.

54) So - bereits vielzitiert - Battis, Die Welt v. 29.3.2020. 